\title{
Drug treatment of pruritus in liver diseases
}

\author{
Authors: Vinod S Hegade, ${ }^{A}$ Stuart FW Kendrick ${ }^{B}$ and David EJ Jones ${ }^{C}$
}

Pruritus (itch) is a common complication of cholestatic liver diseases (CLD). It can be a distressing and debilitating symptom, causing significant impairment in quality of life. Treatment of pruritus in liver diseases can be challenging and requires specific management with early initiation and a step-wise approach using specific drugs. Clinical trials are ongoing with novel agents that demonstrate potential efficacy. Patients with cholestatic pruritus are likely to present to a variety of clinicians who would benefit from medical awareness of available treatment options. In this review, we outline the pharmaceutical agents currently used to treat cholestatic pruritus and provide the evidence base for targeted symptom control of itch in liver diseases. We also highlight recent developments in the pathophysiology of cholestatic pruritus and the emerging novel therapies.

KEYWORDS: Pruritus, cholestasis, primary biliary cirrhosis, liver disease, treatment

\section{Introduction}

The word 'cholestasis', derived from the Greek words 'chole' (meaning bile) and 'stasis' (meaning standing still), refers to impairment of bile formation or bile flow. The impairment could be within the liver (intrahepatic cholestasis or 'tissue cholestasis') or in the bile ducts draining the bile from the liver into the small intestine (extrahepatic cholestasis). Intrahepatic cholestasis usually results either from immunologically mediated destruction of small bile ducts or from defective bile acid transport proteins located within the hepatocytes or cholangiocytes. Extrahepatic cholestasis generally results

\footnotetext{
Authors: ${ }^{\text {A }}$ linical research fellow, Freeman Hospital, Newcastle upon Tyne Hospitals NHS Foundation Trust, Newcastle upon Tyne, UK, and Institute of Cellular Medicine, Faculty of Medical Sciences, Newcastle University, Newcastle upon Tyne, UK; ${ }^{\mathrm{B}}$ consultant hepatologist, Freeman Hospital, Newcastle upon Tyne Hospitals NHS Foundation Trust, Newcastle upon Tyne, UK, and Institute of Cellular Medicine, Faculty of Medical Sciences, Newcastle

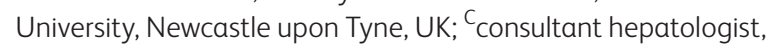
Freeman Hospital, Newcastle upon Tyne Hospitals NHS Foundation Trust, Newcastle upon Tyne, UK, and Institute of Cellular Medicine, Faculty of Medical Sciences, Newcastle University, Newcastle upon Tyne, UK
}

from mechanical obstruction to the bile flow due to stones or strictures. Table 1 gives differential diagnoses of intra- and extrahepatic cholestatic diseases. The underlying mechanism of cholestasis is impairment of hepatobiliary excretion as a result of which normal biliary constituents appear in the systemic circulation. The characteristic biochemical abnormality seen in cholestasis is elevation in serum alkaline phosphatase and gamma-glutamyl transpeptidase.

Pruritus (itch) is a well-known, frequent and distressing symptom of cholestasis. In clinical practice, the most commonly encountered cholestatic liver diseases (CLD) associated with pruritus are primary biliary cirrhosis (PBC), primary sclerosing cholangitis (PSC) and intrahepatic cholestasis of pregnancy. Historically, pruritus has been observed to accompany jaundice, but it is not uncommon to see pruritus as the first manifestation of cholestasis even before the onset of jaundice or other symptoms. There is considerable variation in the frequency and prevalence of pruritus in different cholestatic conditions. For example, it is experienced by up to $80 \%$ of patients with PBC and PSC and by $5-15 \%$ of patients with chronic hepatitis $\mathrm{C}$ at any time during the course of their disease. ${ }^{1}$ It is less common in patients with extrahepatic cholestasis, as in one series pruritus occurred in $17 \%$ of all patients with non-neoplastic obstructive jaundice and in $45 \%$ of patients with neoplastic obstructive jaundice. ${ }^{2}$ Also, pruritus is rare in common liver diseases such as alcohol-related liver diseases and non-alcohol fatty liver diseases. Interestingly, for reasons that are currently unexplained, the severity of pruritus seen in cholestatic conditions has no relationship with the degree of severity of cholestasis, ie patients with similar severities of liver disease and cholestasis can have markedly different degrees of pruritus.

\section{Clinical features}

Clinically, in contrast to pruritus of other systemic conditions (such as uraemia and malignancy), cholestatic pruritus has some unique features. A vast majority of patients report a diurnal variation of itch intensity, with the worst itch in the late evening and early night-time. ${ }^{3}$ Typically, cholestatic pruritus affects limbs, soles of the feet and palms (palmoplantar pruritus) but generalised itch may also occur. It is often exacerbated by psychological stress, heat and contact with certain fabrics such as wool. Premenstrual period, late stages of pregnancy and hormone replacement therapy can also exacerbate the symptom, suggesting a role of female sex hormones in the pathogenesis of cholestatic 
Table 1. Differential diagnoses of cholestatic conditions.

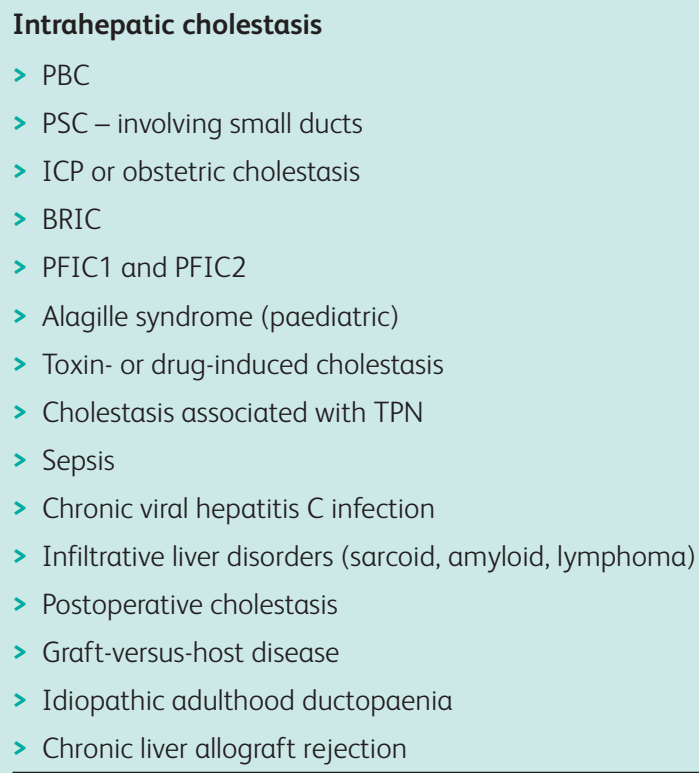

pruritus. ${ }^{1}$ Unlike other causes of pruritus, patients with cholestatic pruritus complain that scratching activity barely relieves their itch. On examination, primary skin lesions are not observed, but vigorous scratching can result in secondary skin lesions such as excoriations, folliculitis (inflammation of hair follicles), prurigo nodularis (hard nodules on the skin) and lichenification (leathery hardening of the skin). The majority of CLD patients with pruritus have mild and tolerable symptoms, but some patients may experience troublesome and frequently agonising itch, which may dramatically reduce their quality of life. In fact, patients with severe intractable pruritus may develop deranged sleep patterns, fatigue, cognitive symptoms, mood changes, anxiety, depression, and even on occasion, suicidal ideations. ${ }^{4}$

In many ways, $\mathrm{PBC}$ is the archetypal cholestatic liver disease, and cholestatic pruritus has been mainly studied in this condition. In PBC, pruritus can develop at any stage of the disease, and once it occurs its severity may diminish over time but it rarely resolves completely without treatment. ${ }^{5}$ Interestingly, recent evidence suggests that the intensity of pruritus in $\mathrm{PBC}$ may be associated with the age at disease presentation. A UK study of over 2,000 PBC patients observed that younger patients are more likely to have higher average levels of itch severity, as the pruritus score was $64 \%$ higher in patients presenting at an age younger than 50 in comparison with those presenting at an age older than $70 .{ }^{6}$ However, the severity of pruritus shows no association with the disease duration, biochemical severity or histological stage of PBC. For example, a PBC patient with an early-stage disease and mildly abnormal liver function tests may experience severe pruritus compared with another PBC patient with endstage liver failure and marked cholestasis but no pruritus. Studies have attempted to characterise perception of itch by PBC patients. In one such study, $35 \%$ of patients described their itch as 'bugs crawling', $62 \%$ reported worsening of

\section{Extrahepatic cholestasis}

> PSC involving extrahepatic (large) bile ducts

$>$ Choledocholithiasis

$>$ Cholangiocarcinoma

$>$ IgG4-associated cholangitis

$>$ Tumours of the pancreatic head

> Hilar lymphadenopathy

$>$ Bile duct adenoma

$>$ Biliary atresia

> Extrahepatic biliary strictures: ischaemic, iatrogenic, infectious (eg AIDS cholangiopathy)

> Parasitic infestation (eg ascaris) itch with heat, and $65 \%$ reported improvement of itch with something cool. ${ }^{7}$

\section{Pathophysiology of pruritus in CLD}

The mechanism of development of pruritus in CLD is complex, multifactorial and remains incompletely understood. There are no proper rodent models for cholestatic pruritus, as experimental cholestatic animals rarely display scratching behaviour. It is suggested that cholestasis triggers the release of pruritogen(s) which accumulate in the plasma and other tissues, stimulate neural itch fibres in the skin and transmit the stimulus to the spinal cord and the brain. Over the last decades, many experimental and clinical studies have attempted to explore the role of putative pruritogens that include histamine, substance P, bile acids and endogenous opioids such as enkephalins, but to date no single substance has been conclusively shown to be the causative pruritogen in cholestasis. More recent studies propose lysophosphatidic acid (LPA - a potent neuronal activator) and autotaxin (an enzyme forming LPA) as mediators in cholestatic pruritus. There is evidence that intradermally injected LPA induces a scratch response in mice, and LPA levels are significantly increased in the sera of cholestatic patients with pruritus compared with those without pruritus. ${ }^{8}$ Also, it has been shown that in contrast to serum bile acids and $\mu$-opioids, serum autotaxin activity strongly correlates with itch intensity. ${ }^{9}$ Although this evidence strongly suggests that LPA and autotaxin play a critical role in cholestatic pruritus, the source of production of autotaxin and the mechanism of rise in its activity in cholestasis remain unexplained. It is proposed that in cholestasis an as-yet unidentified factor ('factor X') that is either biliary excreted or retained in systemic circulation causes upregulation of transcription of autotaxin, which increases circulating autotaxin levels, in turn increasing the levels of LPA, which 
has direct actions on pruritoceptive nerve fibres. ${ }^{4}$ See relevant texts for a more comprehensive review of pathophysiology of cholestatic pruritus. ${ }^{1}$ In summary, the current understanding is that cholestatic pruritus results from a complex interplay of direct or indirect actions of bile acids and/or their metabolites, increased opioidergic neurotransmission, upregulation of autotaxin and increased levels of LPA resulting in triggering of pruritoceptive nerve fibres.

\section{Current treatment of pruritus in CLD}

A patient with cholestatic pruritus needs to be evaluated thoroughly to exclude other systemic and dermatological causes of pruritus. A general inspection of the skin should be conducted for any lesions, and patients with pruritic skin lesions should be referred for dermatology review. The first step in the management of cholestatic pruritus is to exclude biliary obstruction. All patients with cholestasis should have a transabdominal ultrasound (US) as the first radiological investigation, to image the liver and biliary tree. The presence of intrahepatic biliary duct dilatation on US usually suggests biliary obstruction. If diagnosis is uncertain, abdominal computerised tomography (CT) and/or magnetic resonance imaging (MRI)/magnetic resonance cholangiopancreatography (MRCP) may be indicated to characterise obstructive cholestasis. When large bile duct obstruction (eg stones/ strictures) is present, relief of obstruction to bile flow (eg removal of stones/insertion of stent) results in a decrease or disappearance of pruritus.

Ursodeoxycholic acid (UDCA), the most commonly used drug for cholestasis, is not effective in reducing the development or severity of pruritus, and current guidelines do not recommend its use in the treatment of cholestatic pruritus. Similarly, antihistamines do not have any role in the treatment and should not be prescribed. For mild and localised itch, topical treatment with aqueous cream plus $1 \%$ menthol can be used for its emollient and coolant effect. For more severe and generalised itch associated with cholestasis, the American Association for the Study of Liver Diseases (AASLD) and the European Association for the Study of the Liver (EASL) recommend step-wise treatment using colestyramine (cholestyramine), rifampicin (rifampin), naltrexone and sertraline. ${ }^{10,11}$ This is summarised in Table 2 and demonstrated graphically in Fig 1. It is important to note that only colestyramine is approved by regulatory bodies and all other drugs are 'off-label' use. It is a common practice to start with the first-line drug as monotherapy for at least 2-4 weeks (in the absence of side effects). If there is no response, the drug should be stopped and replaced with the next line of therapy. However, if there is a partial or inadequate response, the drug may be continued with the addition of the next line of therapy.

\section{Colestyramine}

Colestyramine is an orally administered non-absorbable anion-exchange resin that is thought to act by removing potential pruritogens (bile salts) from the enterohepatic circulation by binding with them to prevent their reabsorption and thus enhancing faecal excretion. It is the first-line therapy recommended by international guidelines for the treatment of cholestatic pruritus. ${ }^{10,11}$ Four controlled trials and a metaanalysis have confirmed that colestyramine significantly improves cholestatic pruritus. ${ }^{12}$ The recommended initial dose is $4 \mathrm{~g}$ /day, preferably taken in the morning (to remove the pruritogens that accumulate in the gallbladder during overnight

Table 2. Current therapeutic recommendation for the management of pruritus in cholestatic liver diseases.

\begin{tabular}{|c|c|c|c|c|c|}
\hline Class & Drug & Dosage & Route & Comments & Side effects \\
\hline \multirow[t]{2}{*}{ Bile acid resins } & Colestyramine & 4-16 g/day & Oral & Morning dose preferred; & \multirow{2}{*}{$\begin{array}{l}\text { Unpleasant taste } \\
\text { bloating, constipation } \\
\text { and diarrhoea }\end{array}$} \\
\hline & Colesevelam & $\begin{array}{l}3.75 \mathrm{~g} / \text { day in two to } \\
\text { four divided doses }\end{array}$ & Oral & $\begin{array}{l}\text { 2-4 hours before or after } \\
\text { UDCA and other medications }\end{array}$ & \\
\hline $\begin{array}{l}\text { PRX agonist/ } \\
\text { enzyme inducer }\end{array}$ & Rifampicin & 150-600 mg/day & Oral & $\begin{array}{l}\text { Start at } 150 \mathrm{mg} / \mathrm{day} \text {; increase } \\
\text { to } 150 \mathrm{mg} \text { BD if serum } \\
\text { bilirubin >3 mg/dL or } \\
\text { to } 150 \mathrm{mg} \text { TDS if serum } \\
\text { bilirubin < } 3 \mathrm{mg} / \mathrm{dL} \text {; regular } \\
\text { monitoring of blood count } \\
\text { and liver biochemistry }\end{array}$ & $\begin{array}{l}\text { Hepatitis, liver failure } \\
\text { and haemolysis }\end{array}$ \\
\hline \multirow[t]{2}{*}{$\begin{array}{l}\text { Opioid } \mu \text {-receptor } \\
\text { antagonists }\end{array}$} & & & Oral & \multirow[t]{2}{*}{$\begin{array}{l}\text { Regular monitoring of liver } \\
\text { biochemistry }\end{array}$} & \multirow{2}{*}{$\begin{array}{l}\text { Opioid withdrawal-like } \\
\text { reaction: abdominal } \\
\text { pain, high blood } \\
\text { pressure, tachycardia, } \\
\text { goose bumps and } \\
\text { nightmares }\end{array}$} \\
\hline & Naloxone & $0.2 \mu \mathrm{g} / \mathrm{kg} / \mathrm{min}$ & $\begin{array}{l}\text { Continuous } \\
\text { intravenous } \\
\text { infusion for } \\
12-72 \text { hours }\end{array}$ & & \\
\hline SSRI & Sertraline & 75-100 mg/day & Oral & $\begin{array}{l}\text { Start at } 25 \text { mg/day, and } \\
\text { increase gradually }\end{array}$ & $\begin{array}{l}\text { Nausea, dizziness, } \\
\text { diarrhoea, visual } \\
\text { hallucinations and } \\
\text { increased fatigue }\end{array}$ \\
\hline
\end{tabular}

$\mathrm{BD}=$ twice a day; $\mathrm{PXR}=$ pregnane $\mathrm{X}$ receptor; SSRI = selective serotonin re-uptake inhibitor; TDS = three times a day; UDCA = ursodeoxycholic acid. 


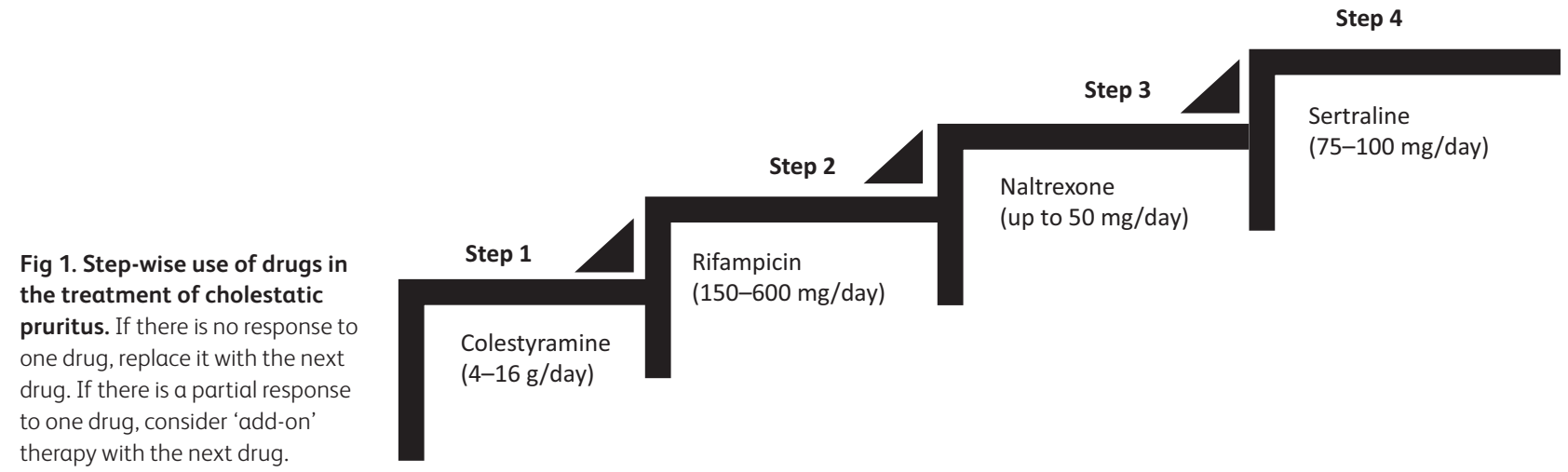

fast and are released in the small intestine after breakfast). Dosage should be gradually increased up to $16 \mathrm{~g} /$ day based on clinical need. Colestyramine should be taken at least 4 hours before or after any other medications, to avoid interference with their intestinal absorption. The main limitation to the use of colestyramine is its unpleasant taste, which can be improved by flavouring the drug with fruit juice. Other adverse effects such as anorexia, constipation, diarrhoea, abdominal discomfort or bloating may also limit its regular use.

In comparison with colestyramine, colesevelam (Cholestagel), a novel anion-binding resin with a seven-fold higher bile acid binding capacity, has fewer side effects and may have better compliance in patients who do not tolerate colestyramine. However, its efficacy is questionable, as a recent small trial showed that colesevelam was not more effective than placebo in reducing the severity of cholestatic pruritus. ${ }^{13}$ Colesevelam should be offered to patients who responded to colestyramine but did not tolerate its side effects.

\section{Rifampicin}

Rifampicin, an enzyme inducer and a commonly used anti-mycobacterial agent, is the recommended second-line agent. The exact mechanism of its anti-pruritic action has been unknown. However, recently, rifampicin was shown to significantly decrease serum autotaxin levels (compared with placebo) and reduce expression of autotaxin in HepG2 cells (in vitro). ${ }^{9}$ The latter effect was pregnane $\mathrm{X}$ receptor (PXR)dependent. These results suggest that the anti-pruritic effect of rifampicin is likely via PXR-agonism mediated downregulation of autotaxin transcription. Four prospective randomised controlled clinical trials and two meta-analyses have confirmed that treatment with rifampicin is safe and leads to complete or partial resolution of pruritus in up to $77 \%$ of patients, as compared with placebo or alternatives. ${ }^{12,14}$ The dosage of rifampicin in these studies was 300-600 mg/day or $10 \mathrm{mg} / \mathrm{kg} /$ day, given orally. The current recommendation is to initiate with $150 \mathrm{mg} /$ day and, based on the clinical need, to titrate the dosage up to a maximum of $600 \mathrm{mg} /$ day. Side effects associated with rifampicin use are nausea, vomiting, diarrhoea, decreased appetite, headaches, fever, rash and flushing. Most of the side effects are transient and resolve on discontinuation of the drug. Side effects that are of serious concern include hepatitis (that may result in hepatic failure), haemolytic anaemia, thrombocytopenia, renal impairment and alteration in drug metabolism. Hepatotoxicity ('rifampicin hepatitis') is most likely to develop in the first 2 months of therapy. An early study reported a $12.5 \%$ incidence of rifampicin hepatitis, and a more recent study reported significant hepatitis in $7.3 \%$ of patients (necessitating liver transplantation in one case). ${ }^{15,16}$ Therefore, in those taking rifampicin, close monitoring of blood counts and liver function tests every fortnight in the first 2 months of therapy and at least once monthly thereafter is strongly recommended; the drug should be stopped if there is a suggestion of hepatotoxicity.

\section{Opioid antagonists}

Selective $\mu$-opioid receptor antagonist agents - naloxone and naltrexone - are recommended as third-line therapy for patients with cholestatic pruritus when the first- and secondline drugs are ineffective or intolerable. It is known that plasma and hepatic levels of endogenous opioids are increased in cholestatic patients and in animal models of cholestasis. ${ }^{17,18}$ More recently, studies have shown that opioid-induced itch is mediated both by an opioid receptor mechanism and by initiating itch through central and peripheral pathways. ${ }^{19}$ Therefore, the likely mechanism of opioid antagonists in reducing itch is by blocking the opioid receptors and by modifying central and peripheral itch and/or pain signalling by influencing the endogenous opioidergic system. Evidence to support the use of either oral (naltrexone and nalmefene) or parenteral (naloxone) opioid antagonists is from a number of prospective studies as well as a recent meta-analysis of five studies that concluded that, compared with the control intervention, opioid antagonists are more likely to significantly reduce cholestatic pruritus. ${ }^{12}$ However, a significant concern with the use of these agents is precipitation of 'opioid withdrawal-like reaction' - a constellation of symptoms characterised by abdominal pain, tachycardia, high blood pressure, goose bumps, nightmares and depersonalisation. ${ }^{10}$ This reaction can be minimised by starting the opioid antagonists at a lower dose and gradually increasing the dose, or by giving intravenous naloxone for 3 days followed by oral naltrexone. The guidelines recommend starting naltrexone at $12.5 \mathrm{mg} /$ day and gradually increasing by $12.5 \mathrm{mg}$ every 3-7 days until amelioration of pruritus (maximum daily dose $50 \mathrm{mg}$ ). Hepatotoxicity is uncommon but it has been 
reported; therefore, regular monitoring of liver biochemistry is recommended. Opioid antagonists are contraindicated in patients with acute hepatitis, liver failure, suppressed pulmonary function, drug addictions and in those receiving opioid-containing medications. An uncommon side effect of long-term use of opioid antagonists is loss of control of pain resulting from other pathologies, leading to a chronic pain syndrome. $^{20}$

\section{Sertraline}

Sertraline, a selective serotonin re-uptake inhibitor (SSRI), commonly prescribed as an antidepressant, is the recommended fourth-line therapy to alleviate cholestatic pruritus. The rationale for its use comes from evidence that the serotonin system modulates nociception and perception of pruritus, and sertraline can influence the endogenous serotoninergic system and modify the central itch and/or pain signalling. Two studies showed that sertraline was well tolerated and moderately effective in reducing the intensity of itch in cholestatic pruritus, and the effect was independent from improvement in depression. ${ }^{21,22}$ The recommended initial dosage is $25 \mathrm{mg}$ /day, increased gradually by $25 \mathrm{mg}$ every $4-5$ days to $75-100 \mathrm{mg} /$ day. Sertraline is usually well tolerated, and uncommon adverse effects include nausea, dizziness, diarrhoea, visual hallucinations and increased fatigue.

\section{Refractory pruritus}

Patients with cholestatic pruritus who remain symptomatic despite maximum drug therapy should be considered for salvage therapy with invasive interventions such as ultraviolet $B$ phototherapy, endoscopic nasobiliary drainage, plasmapheresis and/or molecular adsorbent recirculating system. However, the availability of these treatments is limited to few centres and referral pathways are poorly described. Patients with refractory pruritus should also be offered participation in ongoing clinical trials of novel drug therapy (see below). Finally, patients with cholestatic liver disease and intractable severe itch should be referred for liver transplantation even in the absence of liver synthetic dysfunction. ${ }^{10}$

\section{Novel therapies for cholestatic pruritus}

As seen above, the treatment of cholestatic pruritus is limited by availability of only four classes of drugs that are recommended by guidelines and for which there is a strong evidence base. However, they have poor side-effect profiles and need regular monitoring for adverse events (mainly for hepatotoxicity). Also, treatment can be extremely difficult and challenging in those patients who remain symptomatic despite using various combination therapies. Therefore, there is an unmet need for safer and effective therapies to treat pruritus in cholestasis. Efficient drug discovery has been hindered by a lack of robust knowledge of the molecular mechanisms of cholestatic pruritus. However, recent evidence on autotaxin, LPA and advances in bile acid physiology has initiated the development of novel agents that might represent an alternative to current treatments.

\section{Bile acid transport inhibitors}

Bile acids produced in the liver are secreted into bile which then enters the intestine to facilitate absorption of fat and fat-soluble vitamins. In the ileum, bile acids are reabsorbed to enter the portal circulation and return to the liver. This efficient cycle between the liver and the small intestine, called enterohepatic circulation (EHC), maintains the bile acid pool and ensures that $95 \%$ of bile acids are reabsorbed (Fig 2). A number of bile acid transporter proteins are involved in the EHC, of which apical sodium-dependent bile acid transporter (ASBT) located in the terminal ileum (also called ileal bile acid transporter) has been shown to play a key physiological role in the active absorption of bile acids from the ileum. ${ }^{23}$ Reabsorption of bile acids or other similar but unrecognised pruritogens into the portal circulation can be inhibited by

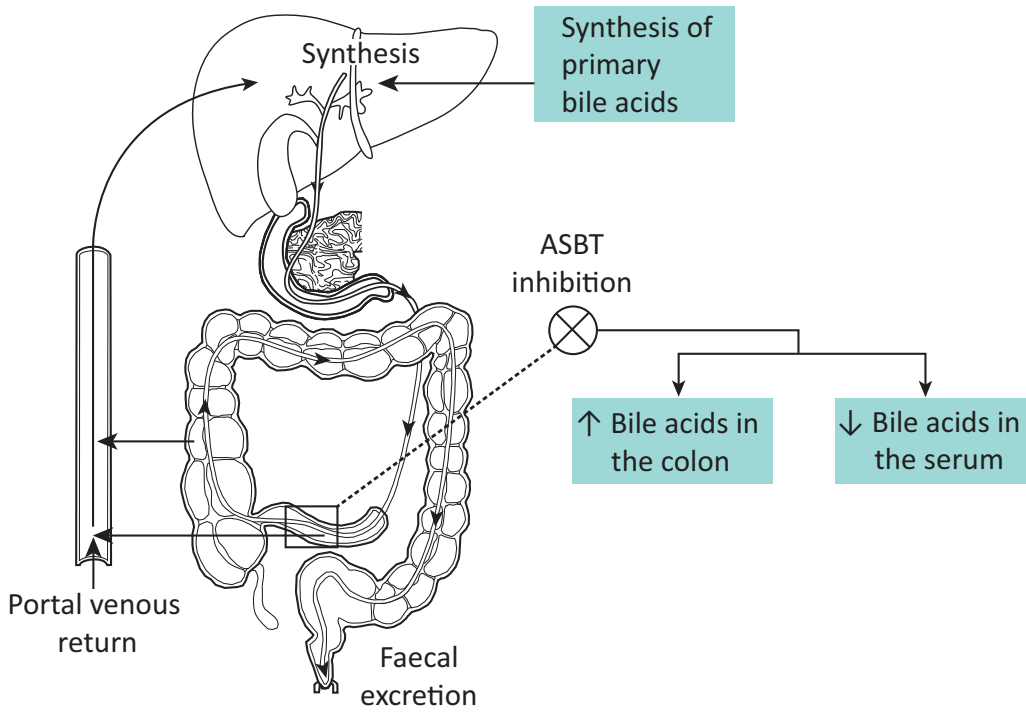

(5\%)
Fig 2. Enterohepatic circulations of bile acids and the effect of inhibition of apical sodium-dependent bile acid transporter on bile acids. ASBT = apical sodium-dependent bile acid transporter. 
blocking the ASBT. Such pharmacological inhibition of ASBT could potentially enhance faecal excretion of pruritogens and reduce their circulating pool, which in turn could reduce symptoms of pruritus (Fig 2). Animal studies have shown that SC-435, a competitive inhibitor of ASBT, increases faecal bile acid outputs (resulting in diarrhoea secondary to bile acid malabsorption) and reduces bile acid levels in the circulation. ${ }^{24}$ Two ongoing multicentre double-blind randomised placebo controlled phase II trials using ASBT inhibitors in patients with $\mathrm{PBC}$ are evaluating the safety, tolerability and efficacy of the drugs (https://clinicaltrials.gov, identifier: NCT01899703 and NCT01904058). If these trials and future large-scale studies confirm their therapeutic efficacy, ASBT inhibitors could become important therapeutic modalities for the treatment of cholestatic pruritus.

\section{Autotaxin inhibitors and LPA receptor blockers}

Experimental evidence has shown that autotaxin and LPA are markedly elevated in the serum of patients with cholestatic pruritus, and that their levels closely correlate with itch intensity. Therefore, autotaxin inhibitors and LPA receptor blockers that are currently being developed as anticancer drugs (to reduce disease progression and metastasis) might represent a novel class of antipruritic agents in cholestatic diseases. Studies have shown that LPA increases cytosolic free calcium levels $\left(\left[\mathrm{Ca}^{2+}\right]_{\mathrm{i}}\right)$ in neuronal cells, and pre-treatment of the neuronal cells with Ki16425 (a specific LPA receptor blocker) significantly reduces the increase in $\left[\mathrm{Ca}^{2+}\right]_{\mathrm{i}} \cdot{ }^{8}$ This preliminary evidence may explain the potential mechanism of LPA receptor blockers, which could be developed as a novel therapeutic agent for treatment of cholestatic pruritus.

As the effects of autotaxin are mainly mediated through enzymatic production of LPA, autotaxin inhibitors that reduce LPA levels could also serve as novel therapeutic options. However, the main concerns with autotaxin-directed therapy include an incomplete understanding of the source of autotaxin production and a lack of understanding of its role in the regenerative capacity and disease progression in cholestasis. These concerns also raise the possibility that autotaxin inhibitors may improve pruritus but at the expense of the worsening of cholestatic biology. ${ }^{4}$ Therefore, future directions for efficient anti-pruritic drug discovery would benefit from efforts to overcome our current lack of knowledge of the 'physiological' role played by autotaxin in CLD.

\section{Conclusion}

Pruritus, a complication of cholestatic liver diseases, is a substantial and challenging clinical problem. The pathophysiology of cholestatic pruritus is only partially understood, and controversies remain with regard to the roles played by proposed pruritogens including bile acids, endogenous opioids and, more recently, autotaxin. No single therapy is effective in the control of pruritus, and often patients need to be treated with different drugs in line with current treatment guidelines. The efficacy of currently available therapeutic agents for cholestatic pruritus is often disappointing, as treatment can be associated with significant side effects and successful amelioration of pruritus is far from universal. Recent evidence on the pathophysiological role of autotaxin and LPA in cholestatic itch, if substantiated further, could potentially help in future drug development strategies. While the results of ongoing trials of bile acid transport inhibitors are eagerly awaited, LPA-receptor blockers and autotaxin inhibitors may represent novel therapeutic strategies for cholestatic pruritus.

\section{References}

1 Kremer AE, Oude Elferink RP, Beuers U. Pathophysiology and current management of pruritus in liver disease. Clin Res Hepatol Gastroenterol 2011;35:89-97.

2 McPhedran NT, Henderson RD. Pruritus and jaundice. Can Med Assoc J 1965;92:1258-60.

3 Kremer AE, Beuers U, Oude-Elferink RP, Pusl T. Pathogenesis and treatment of pruritus in cholestasis. Drugs 2008;68:2163-82.

4 Jones DE. Pathogenesis of cholestatic itch: old questions, new answers, and future opportunities. Hepatology 2012;56:1194-6.

5 Talwalkar JA, Souto E, Jorgensen RA et al. Natural history of pruritus in primary biliary cirrhosis. Clin Gastroenterol Hepatol 2003;1:297-302.

6 Carbone M, Mells GF, Pells G et al. Sex and age are determinants of the clinical phenotype of primary biliary cirrhosis and response to ursodeoxycholic acid. Gastroenterology 2013;144:560-9.

7 Rishe E, Azarm A, Bergasa NV. Itch in primary biliary cirrhosis: a patient's perspective. Acta Derm Venereol 2008;88:34-7.

8 Kremer AE, Martens JJ, Kulik W et al. Lysophosphatidic acid is a potential mediator of cholestatic pruritus. Gastroenterology 2010;139:1008-18.

9 Kremer AE, van Dijk R, Leckie P et al. Serum autotaxin is increased in pruritus of cholestasis, but not of other origin, and responds to therapeutic interventions. Hepatology 2012;56:1391-400.

10 Lindor KD, Gershwin ME, Poupon R et al. Primary biliary cirrhosis. Hepatology 2009;50:291-308.

11 European Association for the Study of the Liver. EASL clinical practice guidelines: management of cholestatic liver diseases. J Hepatol 2009;51:237-67.

12 Tandon P, Rowe BH, Vandermeer B et al. The efficacy and safety of bile acid binding agents, opioid antagonists, or rifampin in the treatment of cholestasis-associated pruritus. Am J Gastroenterol 2007;102:1528-36.

13 Kuiper EM, van Erpecum KJ, Beuers U et al. The potent bile acid sequestrant colesevelam is not effective in cholestatic pruritus: results of a double-blind, randomized, placebo-controlled trial. Hepatology 2010;52:1334-40.

14 Khurana S, Singh P. Rifampin is safe for treatment of pruritus due to chronic cholestasis: a meta-analysis of prospective randomizedcontrolled trials. Liver Int 2006;26:943-8.

15 Bachs L, Parés A, Elena M, Piera C, Rodés J. Effects of long-term rifampicin administration in primary biliary cirrhosis. Gastroenterology 1992;102:2077-80.

16 Prince MI, Burt AD, Jones DE. Hepatitis and liver dysfunction with rifampicin therapy for pruritus in primary biliary cirrhosis. Gut 2002;50:436-9.

17 Swain MG, Rothman RB, Xu H et al. Endogenous opioids accumulate in plasma in a rat model of acute cholestasis. Gastroenterology 1992;103:630-5.

18 Bergasa NV, Vergalla J, Swain MG, Jones EA. Hepatic concentrations of proenkephalin-derived opioids are increased in a rat model of cholestasis. Liver 1996;16:298-302.

19 Greaves MW. Pathogenesis and treatment of pruritus. Curr Allergy Asthma Rep 2010;10:236-42.

20 McRae CA, Prince MI, Hudson M et al. Pain as a complication of use of opiate antagonists for symptom control in cholestasis. Gastroenterology 2003;125:591-6. 
21 Browning J, Combes B, Mayo MJ. Long-term efficacy of sertraline as a treatment for cholestatic pruritus in patients with primary biliary cirrhosis. Am J Gastroenterol 2003;98:2736-41.

22 Mayo MJ, Handem I, Saldana $S$ et al. Sertraline as a first-line treatment for cholestatic pruritus. Hepatology 2007;45:666-74.

23 Pellicoro A, Faber KN. Review article: the function and regulation of proteins involved in bile salt biosynthesis and transport. Aliment Pharmacol Ther 2007;26(Suppl 2):149-60.
24 Dawson PA, Haywood J, Craddock AL et al. Targeted deletion of the ileal bile acid transporter eliminates enterohepatic cycling of bile acids in mice. J Biol Chem 2003;278:33920-7.

Address for correspondence: Dr V Hegade, Second Floor, Institute of Cellular Medicine, Faculty of Medical Sciences, Newcastle University, Newcastle upon Tyne NE2 4HH, UK. Email: vinod.hegade@ncl.ac.uk $11^{\text {th }}$ BAD RCP MEDICAL DERMATOLOGY MEETING Royal College of Physicians, London Thursday $14^{\text {th }}$ January 2016 9:00-17:00

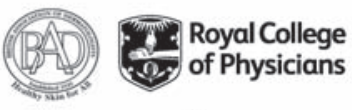

We invite you to register for the $11^{\text {th }}$ Medical Dermatology Meeting. This year's theme is Dermatology and Genetics. The meeting consists of a series of invited, clinically orientated lectures by Dermatologists and Geneticists. Talks will include Ehlers-Danlos Syndrome, Tuberous Sclerosis Complex, Neurofibromatosis and Familial Melanoma. The Parkes Weber Lecture 'Saving the Singing Detective' will be given by Professor Jonathan Barker. Trainees have the opportunity to submit an abstract of a case which illustrates the interface between Skin disease and Genetics for possible poster or oral presentation and publication in the British Journal of Dermatology.

Please email conference@bad.org.uk for further information including registration details and to request an abstract submission form.

British Society for Medical Dermatology

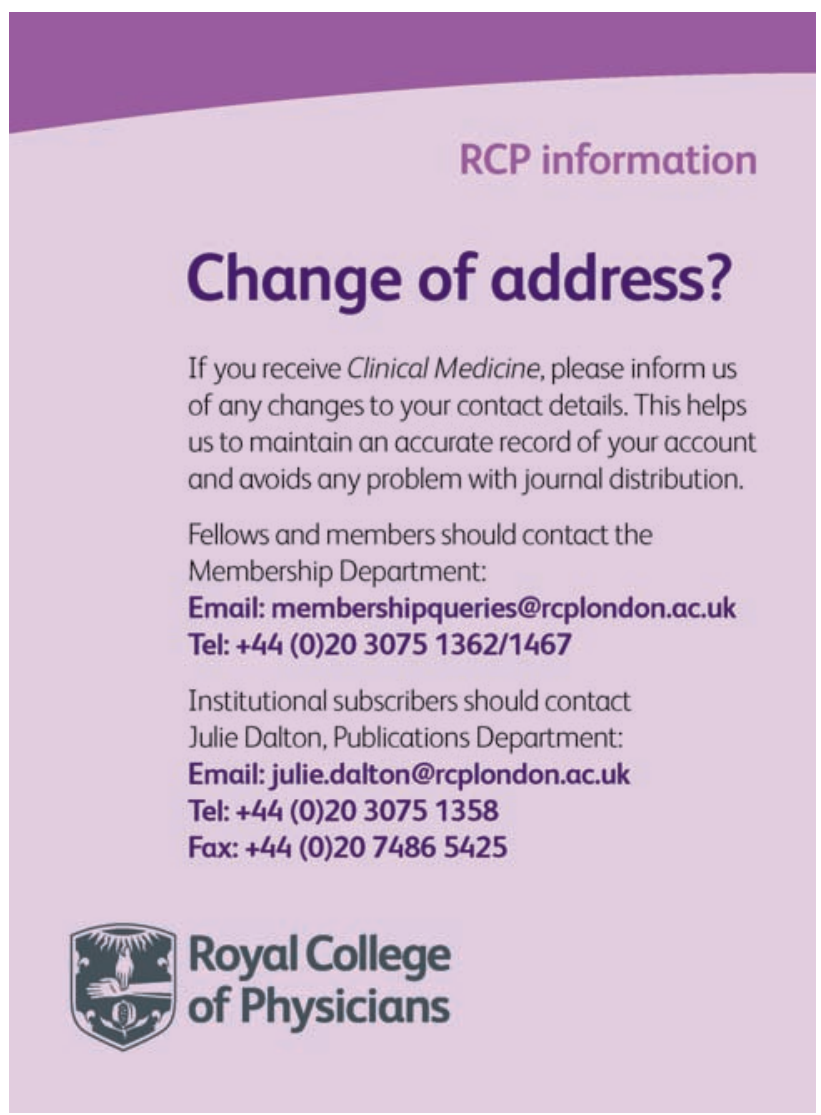

